
I. L. Nikonov ${ }^{1,2}$,
D. S. Kopchuk ${ }^{1,2}$,
A. F. Khasanov ${ }^{1,2}$, A.P. Krinochkin ${ }^{1,2}$, E. S. Starnovskaya ${ }^{1}$, Ya. K. Shtaiz ${ }^{1}$, M. I Savchuk ${ }^{1}$, O.S. Tanya ${ }^{1}$, G. V. Zyryanov ${ }^{1,2}$, V. L. Rusinov ${ }^{1,2}$, O.N. Chupakhin ${ }^{1,2}$
${ }^{1}$ Ural Federal University, 19 Mira St., Ekaterinburg 620002, Russian Federation
${ }^{2}$ I. Ya. Postovskii Institute of Organic Synthesis, Ural Branch of Russian Academy of Sciences, 22 Kovalevskaya St./20 Akademicheskaya St., Ekaterinburg 620990, Russian Federation E-mail:dkopchuk@mail.ru

\title{
Two mutually complementary synthetic approaches towards 3-substituted 3,4-disubstituted and 1-(2-pyridyl)-substituted isoquinolines
}

Two mutually complementary synthetic approaches towards 3- and 3,4-disubstituted 1-(2-pyridyl) isoquinolines were studied. The aryne-based method was successfully used for the obtaining of the corresponding the 3-cyano-1-(2-pyridyl)isoquinolines in one step/pot reaction, while it is unacceptable for the obtaining of other 1-(2-pyridyl)isoquinolines. The enamine-based approach was successfully applied for the synthesis of other 1-(2-pyridyl)isoquinolines, while it was unacceptable for the obtaining of 3-cyano-1-(2-pyridyl)isoquinolines.

Keywords: 1,2,4-triazines; arynes; enamines; isoqunolines; aza-Diels-Alder reaction; dominotransfrormation.

Received: 08.09.2018. Accepted: 19.10.2018. Published: 31.10.2018.

(C) Nikonov I. L., Kopchuk D. S., Khasanov A. F., Krinochkin A. P., Starnovskaya E. S.,

Shtaiz Ya. K., Savchuk M. I, Tanya 0. S., Zyryanov G. V., Rusinov V.L., Chupakhin 0. N., 2018

\section{Results and Discussion}

Aryne intermediates, generated in situ, are currently attracting more and more attention from the point of view of their use in organic synthesis, since practically useful products of various purposes can be obtained [1-3]. Recently, we have demonstrated the possibilities of their successful use in reactions with substituted 1,2,4-triazines for obtaining both the expected aza-Diels-Alder reaction products, namely the corresponding isoquinolines, and the domino transformations, for example, 10-(1H-1,2,3-triazol-1-yl) pyrido $[1,2-a]$ indoles. The direction of the reaction depends on the nature of the 1,2,4-triazines (or aryns) introduced into the composition of the substituents [4].

This article analyzes the two synthetic approaches we have developed for the synthesis of 1- (2-pyridyl) isoquinolines with different substituents in the $\mathrm{C} 3$ and $\mathrm{C} 4$ positions, which are of interest, in particular, as ligands for transition metal cations [5], as well as from the point of view of creating OLED [6]. 
Thus, the reaction of 3-(2-pyridyl) -1,2,4-triazines 1 a, having an aromatic substituent or a hydrogen atom at the $\mathrm{C} 5$ position, with aryne results in the corresponding pyrido [1,2-a] indoles 2 [7] (scheme 1), while the synthesis of target 1-(2-pyridyl) isoquinolines $\mathbf{3 a}$ in this way is impossible. To solve this problem, we developed an alternative synthetic approach, which was based on the use of 3-(2-pyridyl)-1,2,4-triazines $\mathbf{1}$ as starting compounds. The approach involves the preparation of 5,6,7,8-tetrahydroisoquinolines $4 \mathbf{a}$ as a result of the reaction of aza-Diels-Alder (Boger) with enamine followed by oxidative aromatization of the isoquinoline system [8]. 1-Morpholinocyclohexene was used as a dienophile for the first stage. Subsequent aromatization using DDQ as an oxidizing agent made it possible to successfully synthesize isoquinolines $\mathbf{3 a}$.

It should be noted that in the reaction of 3-(2-pyridyl)-1,2,4-triazine-5-carbonitriles $\mathbf{1 b}$ with arynes, the corresponding isoquinolines $\mathbf{3 b}$ were also obtained as main products, whereas the products of domino transformation were the minor products (the yield is not more than 3\%) [9].

We also investigated the possibility of obtaining isoquinolines $\mathbf{3 b}$ as a result of two-stage synthesis through the preparation of tetrahydroisoquinolines $\mathbf{4 b}$. The first step was performed by the same procedure as in the case of synthesis $\mathbf{4 a}$, and afforded the compound $\mathbf{4 b}$. However, subsequent aromatization of tetrahydrocyanoisoquinoline under various conditions, such as boiling in $o$-xylene or 4-chlorotoluene with oxidants, such as DDQ or chloranil, as well as prolonged boiling in the same high-boiling solvents in the presence of $\mathrm{Pd} / \mathrm{C}$ did not lead to the formation of the desired isoquinolines $\mathbf{3 b}$. In all cases the initial tetrahydroisoquinoline $\mathbf{4 b}$ was isolated. Thus, the application of this method is not acceptable for the obtaining the target 3-cyanoisoquinolines $\mathbf{3 b}$.

Thus, it was demonstrated that two mutually complementary synthetic methodologies can be used to synthesize 3-aryl, 3,4-diaryl-, as well as 3-cyano-1-(2-pyridyl)isoquinolines. Thus, in the case of $\mathrm{R}=$ $\mathrm{CN}$ (Scheme 1), the synthesis using aryne intermediates makes it possible to efficiently obtain the corresponding isoquinolines $3 \mathbf{b}$, while the method based of the preparation tetrahydroisoquinolines $\mathbf{4 b}$ does not allow this because of the impossibility of subsequent aromatization by using the common methods. At the same time, in the

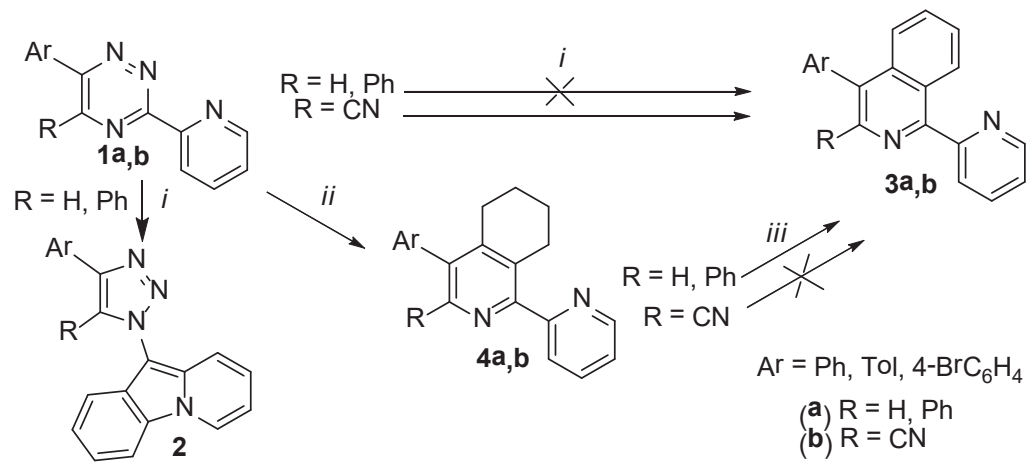

Scheme 1. Reagents and conditions: i) Anthranilic acid, isoamylnitrile, toluene - 1,4-dioxane

(4: 1), boiling, $1.5 \mathrm{~h}$; ii) 1-morpholinocyclohexene, without solvent, $200^{\circ} \mathrm{C}, 4 \mathrm{~h}$; iii) $\mathrm{DDQ}$, $o$-xylene, $143^{\circ} \mathrm{C}, 10 \mathrm{~h}$ 
case of $\mathrm{R}=\mathrm{H}$ or $\mathrm{Ar}$, the opposite situation is observed: the synthesis of isoquinolines 3a is possible with the use of a two-step pathway by using the corresponding enam- ine, and in the case of using aryl intermediates, the reaction leads mainly to rearrangement products $\mathbf{2}$.

\section{Experimental}

NMR ${ }^{1} \mathrm{H}$ and ${ }^{13} \mathrm{C}$ spectra were recorded on the spectrometer "Bruker-Avance-400" (400 MHz), internal standard is $\mathrm{SiMe}_{4}$. The melting points were measured on the "Boetius" device. Mass spectra (type of ionization is electrospray) were recorded on the device of series "MicrOTOF-Q II" of "Bruker Daltonics" (Bremen, Germany). Elemental analyses were performed on CHN analyzer PE 2400, series II by Perkin Elmer.

\section{Acknowledgements}

This work was supported by the Russian Science Foundation (Reference \# 18-13-00365).

\section{References}

1. Yoshida S, Hosoya T. The Renaissance and Bright Future of Synthetic Aryne Chemistry. Chem Lett. 2015;44(11):1450-60. DOI: 10.1246/cl.150839.

2. Wu D, Ge H, Liu SH, Yin J. Arynes in the synthesis of polycyclic aromatic hydrocarbons. RSC Advances. 2013;3(45):22727-38. DOI:10.1039/C3RA43804J.

3. Miyabe H. Synthesis of Oxygen Heterocycles via Aromatic C-O Bond Formation Using Arynes. Molecules. 2015;20(7):12558.

4. Kopchuk DS, Nikonov IL, Khasanov AF, Giri K, Santra S, Kovalev IS, et al. Studies on the interactions of 5-R-3-(2-pyridyl)-1,2,4-triazines with arynes: inverse demand aza-Diels - Alder reaction versus aryne-mediated domino process. Org Biomol Chem. 2018;16(28):5119-35. DOI: 10.1039/C8OB00847G.

5. Mikata Y, Yamanaka A, Yamashita A, Yano S. Isoquinoline-Based TQEN Family as TPEN-Derived Fluorescent Zinc Sensors. Inorganic Chemistry. 2008;47(16):7295301. DOI: $10.1021 /$ ic8002614.

6. Tsuboyama A, Iwawaki H, Furugori M, Mukaide T, Kamatani J, Igawa S, et al. Homoleptic Cyclometalated Iridium Complexes with Highly Efficient Red Phosphorescence and Application to Organic Light-Emitting Diode. J Am Chem Soc. 2003;125(42):12971-9. DOI: 10.1021/ja034732d.

7. Nikonov IL, Kopchuk DS, Kovalev IS, Zyryanov GV, Khasanov AF, Slepukhin PA, et al. Benzyne-mediated rearrangement of 3-(2-pyridyl)-1,2,4-triazines into 10-(1H1,2,3-triazol-1-yl)pyrido[1,2-a]indoles. Tetrahedron Lett. 2013;54(48):6427-9. DOI: https://doi.org/10.1016/j.tetlet.2013.09.042.

8. Kopchuk DS, Kovalev IS, Khasanov AF, Zyryanov GV, Slepukhin PA, Rusinov VL, et al. A rational protocol for the synthesis of 1-(2-pyridyl)isoquinolines. Mendeleev Commun. 2013;23(3):142-4. DOI: 10.1016/j.mencom.2013.05.007.

9. Kopchuk DS, Nikonov IL, Zyryanov GV, Kovalev IS, Rusinov VL, Chupakhin ON. Preparation of 3-Cyano-1-(2-Pyridyl)Isoquinolines by Using Aryne Intermediates. Chem Heterocycl Compd. 2014;50(6):907-10. DOI: 10.1007/s10593-014-1545-9. 